This article was downloaded by: [National Taiwan University]

On: 3 September 2009

Access details: Access Details: [subscription number 905688740]

Publisher Taylor \& Francis

Informa Ltd Registered in England and Wales Registered Number: 1072954 Registered office: Mortimer House, 37-41 Mortimer Street, London W1T 3JH, UK

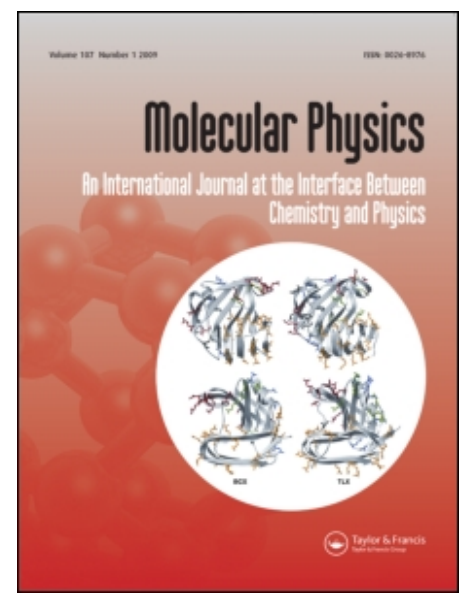

Molecular Physics

Publication details, including instructions for authors and subscription information:

http://www.informaworld.com/smpp/title content=t713395160

\title{
Intermolecular nuclear relaxation and RISM pair-correlation function in liquids
}

Lian-Pin Hwang ab

${ }^{a}$ Department of Chemistry, National Taiwan University, Taipei, Taiwan, Republic of China ${ }^{\mathrm{b}}$ Institute of Biological Chemistry, Academic Sinica, Nankang, Taipei, Taiwan, Republic of China

Online Publication Date: 10 April 1984

To cite this Article Hwang, Lian-Pin(1984)'Intermolecular nuclear relaxation and RISM pair-correlation function in liquids',Molecular Physics, $51: 5,1235-1241$

To link to this Article: DOI: $10.1080 / 00268978400100791$

URL: http://dx.doi.org/10.1080/00268978400100791

\section{PLEASE SCROLL DOWN FOR ARTICLE}

Full terms and conditions of use: http://www.informaworld.com/terms-and-conditions-of-access.pdf

This article may be used for research, teaching and private study purposes. Any substantial or systematic reproduction, re-distribution, re-selling, loan or sub-licensing, systematic supply or distribution in any form to anyone is expressly forbidden.

The publisher does not give any warranty express or implied or make any representation that the contents will be complete or accurate or up to date. The accuracy of any instructions, formulae and drug doses should be independently verified with primary sources. The publisher shall not be liable for any loss, actions, claims, proceedings, demand or costs or damages whatsoever or howsoever caused arising directly or indirectly in connection with or arising out of the use of this material. 


\title{
Intermolecular nuclear relaxation and RISM pair-correlation function in liquids
}

\author{
by LIAN-PIN HWANG \\ Department of Chemistry, National Taiwan University, \\ Taipei, Taiwan, Republic of China, and \\ Institute of Biological Chemistry, Academia Sinica, \\ Nankang, Taipei, Taiwan, Republic of China
}

(Received 15 August 1983 ; accepted 22 October 1983)

\begin{abstract}
The intermolecular proton-proton pair-correlation function (pcf) obtained from the reference interaction site model (RISM) is used as the equilibrium distribution and an effective force in spin relaxation theory. Agreement with experiments on proton dipolar relaxation in liquid acetonitrile, chloroform and ethane is obtained in this analysis. For molecules with non-uniformly distributed spin sites the pair-correlation effect may not be neglected in the treatment of relaxation theory. Aspects concerning uniform pair-distribution and orientational correlations in spin relaxations are briefly discussed.
\end{abstract}

\section{INTRODUCTION}

Intermolecular proton relaxation processes of a molecule in a liquid occur mainly as a result of dipolar interactions [1]. Because the motion modulates the magnetic interactions of the nuclei, the characteristic nuclear relaxation times are related to the motional freedom and spin site-site distribution between molecules. Theoretical treatments of intermolecular relaxation are restricted mostly to monoatomic liquids since the interacting nuclei are assumed to be at the centres of the spin bearing molecules. The correction of the off centre effect has been considered by Hubbard [2]. However, pair-correlation effects in liquids were still neglected.

Starting from the Smoluchowski equation, Hwang and Freed [3] have shown how to correct the time correlation functions and spectral densities needed for spin relaxation by including pair-correlation effects in the dynamical time-evolution of translational diffusion. They have shown that even a simple hard sphere model could lead to an enhanced relaxation rate in comparison with the result using a uniform pair distribution. Moreover, unlike the independent diffusion model treated by Abragam [1], they have considered the proper boundary condition in which the molecular spheres do not penetrate each other even in the course of a trajectory. A recent study in the theoretical formulation of intermolecular relaxation invoking the molecular pef was made by Zeidler [4]. Both radial and orientational corrections have been taken into account in the initial equilibrium distribution whereas for the conditional probability, the independent diffusion model is still assumed in his analysis. Analogous 
effects, taking into account the impenetrability and hard sphere pcf from Monte Carlo simulations in binary liquid mixtures, are discussed by Albrand et al. [5] in their treatment of proton relaxation in paramagnetic solutions.

The success of theories like the RISM equation [6-9] suggests a better understanding of structures of non-associated liquids. The resultant site-site pcf rather than the centre of mass pcf would therefore provide implicitly a solution to correct the off centre effect in the treatment of intermolecular relaxation rates. To avoid further complication of the problem we have neglected the orientational correlations and applied the orientationally averaged intermolecular proton-proton pcf, obtained from RISM calculation, to the evaluation of relaxation rates. The comparison with experiments and further development are also discussed.

\section{THEORY}

The intermolecular proton-proton relaxation rate $1 / T_{1}$ may be obtained by the expression [1]

$$
1 / T_{1}=(4 \pi / 5) \gamma_{\mathrm{H}}{ }^{4} \hbar^{2} I(I+1)[J(\omega)+4 J(2 \omega)],
$$

where $\gamma_{\mathbf{H}}$ is the gyromagnetic ratio of the proton, $I=1 / 2$ is the proton spin quantum number, and $J(\omega)$ is the spectral density defined by $[1,3]$

$$
J(\omega)=2 \operatorname{Re} \int_{0}^{\infty} \exp (i \omega t) G(t) d t
$$

where $G(t)$ is the time-correlation function for dipolar interaction between spins 1 and 2 . It may be expressed by

$$
G(t)=(5 / 4 \pi) n_{\mathrm{H}} \int d^{3} r_{0} \int d^{3} r D_{0, m}{ }^{* 2}\left(\Omega_{0}\right) D_{0, m}{ }^{2}(\Omega) p\left(\mathbf{r}_{0} \mid \mathbf{r}, t\right) g\left(r_{0}\right) /\left(r_{0}{ }^{3} r^{3}\right) .
$$

In (1) and (2), $n_{\mathrm{H}}$ is the average number density of proton spins and $p\left(\mathbf{r}_{\mathbf{0}} \mid \boldsymbol{r}, t\right)$ is the conditional probability for the relative diffusion of spins 1 and 2 ; i.e., given these spins are separated by $r_{0}$ at $t=0$, it gives the probability they are separated by $r$ at time $t$. Also, $D_{n, m}{ }^{L}(\Omega)$ is the Wigner rotation matrix element, depending on the sets of Eulerian angles $\Omega_{0}$ and $\Omega$ at times zero and $t$ which fix the coordinate system between the intermolecular vector $r$ and the laboratory coordinate frame. We now approximate $p\left(\mathbf{r}_{\mathbf{0}} \mid \mathbf{r}, t\right)$ as the solution of the Smoluchowski equation, i.e.

$$
\frac{\partial p\left(\mathbf{r}_{0} \mid \mathbf{r}, t\right)}{\partial t}=D \nabla \cdot\left[\nabla p\left(\mathbf{r}_{0} \mid \mathbf{r}, t\right)+\nabla u(r) p\left(\mathbf{r}_{0} \mid \mathbf{r}, t\right) / k T\right]
$$

with the initial condition

$$
\lim _{t \rightarrow 0} p\left(\mathbf{r}_{0} \mid \mathbf{r}, t\right)=\delta\left(\mathbf{r}-\mathbf{r}_{0}\right)
$$

and boundary conditions

and

$$
\left.\frac{\partial p\left(\mathbf{r}_{0} \mid \mathbf{r}, t\right)}{\partial r}\right|_{r=d}=0
$$

$$
\lim _{r \rightarrow \infty} p\left(\mathbf{r}_{0} \mid \mathbf{r}, t\right)=0
$$


where $d$ is the closest approaching distance between intermolecular spin sites and taken to be the van der Waals diameter of protons. In equation (4), $D$ is the mutual diffusion coefficient and is assumed to equal twice the self diffusion coefficient of the spin bearing molecule. Also, $u(r)$ is the potential of averaged forces between the intermolecular proton sites and is assumed, for simplicity, to depend only upon the radial separation but not molecular orientations. Then we may obtain $u(r)$ from the pcf, $g(r)$, i.e.

$$
\ln g(r)=-u(r) / k T
$$

so that one has an effective force

$$
F(r)=-(\nabla u(r)) / k T=\nabla(\ln g(r)) .
$$

Equation (9), when incorporated into (4) implies that, in the limit $t \rightarrow \infty$, $p\left(\mathbf{r}_{\mathbf{0}} \mid \boldsymbol{r}, t\right)$ will yield the equilibrium $g(r)$, while, for finite times, $F(r)$ is the driving force acting to restore this equilibrium. Here we treat $g(r)$ as the protonproton pcf obtained from RISM calculations.

Now, equations (2) and (3) may be evaluated by means of the finite different method to solve the Smoluchowski equation. The detailed numerical method is discussed elsewhere [3] and will not be given here.

\section{Results AND DISCUSSIONS}

We have solved the RISM integral equation for the liquid models of pure acetonitrile, chloroform and ethane using Lowden's FORTRAN program [10]. The van der Waals diameter of protons, $\sigma_{\mathrm{H}}$, is taken as $2.4 \AA$ [11] for all the RISM molecules given below. The RISM equation provides an approximate method for calculating atom-atom intermolecular pcfs including the protonproton pcf needed in the calculation of relaxation rates.

\subsection{Liquid acetonitrile}

The RISM picture of an acetonitrile molecule is shown in figure 1. The molecule is assumed to be rigid. Reasonable values of the RISM parameters are utilized in the determination of neutron and X-ray scattering structure factors by Hsu and Chandler [7], e.g.

$$
\sigma_{\mathrm{N}}=3.3 \AA, \quad \sigma_{\mathrm{C}(\mathrm{CN})}=3.4 \AA \quad \text { and } \quad \sigma_{\mathrm{C}\left(\mathrm{CH}_{3}\right)}=3.0 \AA
$$

as the van der Waals diameters for the nitrogen atoms and the carbon atoms associated with the $\mathrm{CN}$ and $\mathrm{CH}_{3}$ groups respectively. Tetrahedral bonding of the methyl carbon atom is assumed. In addition, the following intramolecular atom-atom lengths are incorporated in the RISM calculations.

and

$$
\begin{aligned}
& L_{14}=L_{24}=L_{34}=1 \cdot 10 \AA, \\
& L_{15}=L_{25}=L_{35}=2.11 \AA, \\
& L_{16}=L_{26}=L_{36}=3.16 \AA, \\
& L_{45}=1.47 \AA, \\
& L_{46}=2.62 \AA \\
& L_{56}=1.16 \AA .
\end{aligned}
$$




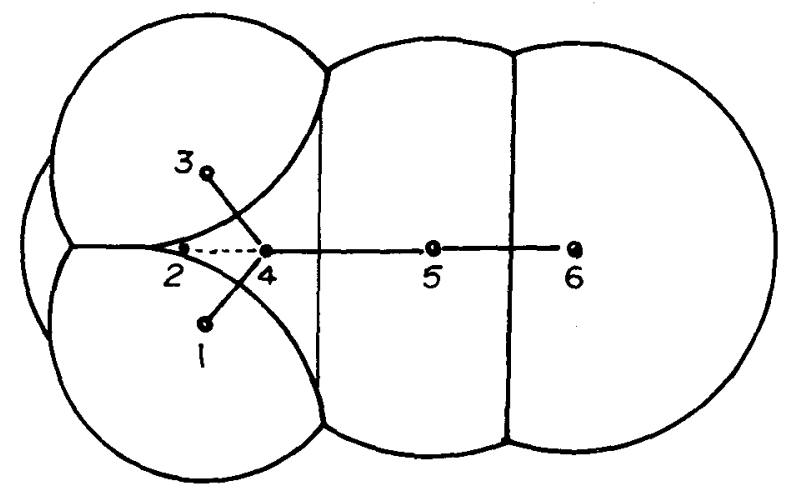

Figure 1. The RISM model for acetonitrile. Atoms 1 to 3 are hydrogens, 4 and 5 are carbons and 6 is the nitrogen. The model parameters are given in the text.

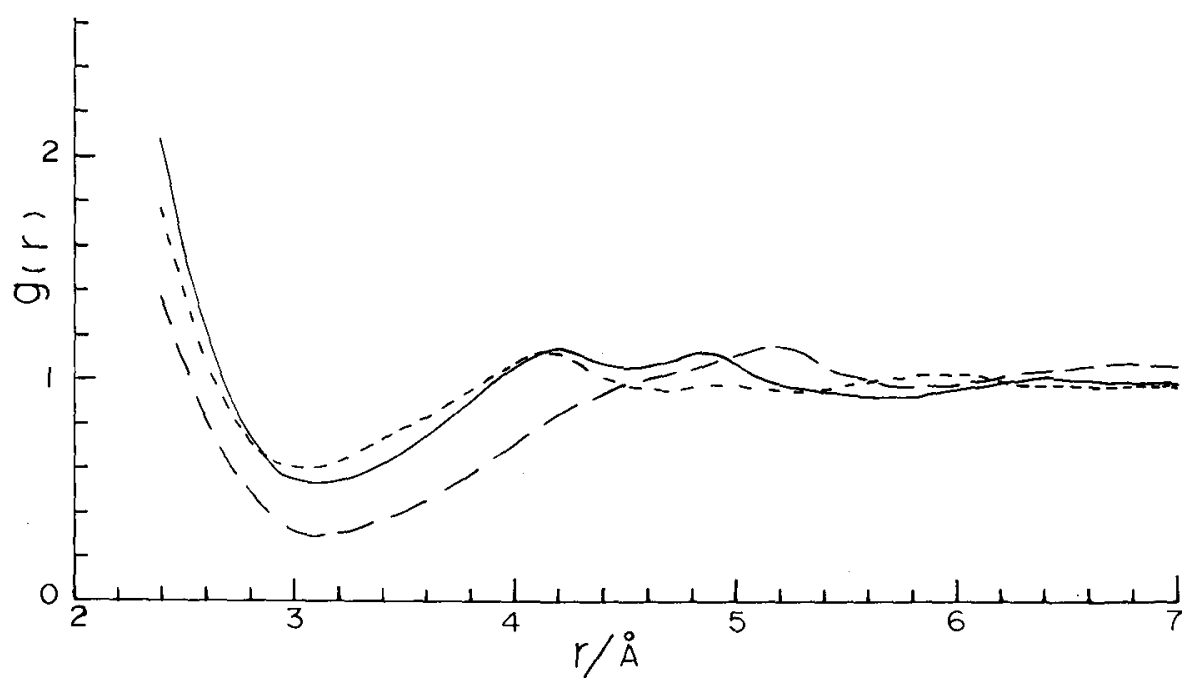

Figure 2. Intermolecular proton-proton pair-correlation functions for liquid acetonitrile, chloroform and ethane. These functions were determined by solving the RISM equation with the molecular parameters given in the text. The dashed curve is for acetonitrile, the broken curve for chloroform and the solid curve for ethane.

The molecular density of the liquid was taken to be $\rho=0.011531 \AA^{-3}$ at $20^{\circ} \mathrm{C}$ and $1 \mathrm{~atm}$. The proton-proton pcf obtained is shown in figure 2. By taking advantage of the experimental diffusion coefficient of acetonitrile [12], the calculated longitudinal relaxation rate at $25^{\circ} \mathrm{C}$ and $25 \mathrm{MHz}$ yields a value $2.3 \times 10^{-2} \mathrm{~s}^{-1}$ that is in good agreement with the observed value $\left(2.3 \times 10^{-2} \mathrm{~s}^{-1}\right)$ [13]. If a value for $\sigma_{\mathrm{H}}$ of $2 \cdot 2 \AA$ is used, the result shows a 6 per cent increase in the relaxation rates.

\subsection{Liquid chloroform}

The parameters used in the RISM model for $\mathrm{CHCl}_{3}[8]$ in the study of neutron scattering experiments are $\sigma_{\mathrm{Cl}}=3.4 \AA$ and $\sigma_{\mathrm{C}}=3 \cdot 0 \AA$. The structure of the chloroform molecule is assumed to be rigid with tetrahedral bonding 
with bond lengths given by $L_{\mathrm{CCl}}=1.76 \AA$ and $L_{\mathrm{CH}}=1 \cdot 10 \AA$. The protonproton pcf obtained for liquid chloroform at $25^{\circ} \mathrm{C}$ is shown in figure 2 .

The intermolecular proton-proton relaxation of liquid chloroform has been studied over the temperature range from $-18^{\circ} \mathrm{C}$ to $47^{\circ} \mathrm{C}$ by Bender and Zeidler [14]. The experimental quantity $D /\left(2 T_{1} n_{\mathrm{H}}\right)=3 \cdot 0 \times 10^{-29} \mathrm{~cm}^{5} \mathrm{~s}^{-2}$ seems to be temperature independent. By varying the densities in the RISM calculation of the pcf, the corresponding calculated values are $2.7 \times 10^{-29}$ to $2.8 \times 10^{-29} \mathrm{~cm}^{5} \mathrm{~s}^{-2}$ over the temperature range from $-18^{\circ} \mathrm{C}$ to $47^{\circ} \mathrm{C}$. The small variation in the calculated values may be explained by the fact that liquid chloroform is treated as a normal non-associated liquid.

\subsection{Liquid ethane}

The staggered conformer of ethane, shown in figure 3 is treated as our RISM molecule. The C-C bond length is taken as $1.54 \AA[11]$ and the $\mathrm{C}-\mathrm{H}$ bond length as $1 \cdot 10 \AA$. 'Thus, for ethane we have the following intramolecular atom-atom lengths

and

$$
\begin{aligned}
& L_{18}=L_{28}=L_{38}=L_{54}=L_{64}=L_{74}=2.17 \AA, \\
& L_{16}=L_{17}=L_{25}=L_{27}=L_{35}=L_{36}=2.50 \AA \\
& L_{12}=L_{23}=L_{31}=L_{56}=L_{67}=L_{75}=1.80 \AA
\end{aligned}
$$

$$
L_{26}=L_{37}=L_{51}=3.08 \AA \text {. }
$$

Also, the van der Waals diameter of the $\mathrm{C}$ atom is taken as $3.0 \AA$.

The RISM equation was solved for liquid ethane with a molecular density $\rho=0.01314 \AA^{-3}$ at $90.71 \mathrm{~K}$. The resulting proton-proton pcf is shown in figure 2. The comparison of calculated relaxation rates with experimental results [15], in three different fields giving Larmor frequencies of 3, 30 and $73 \mathrm{MHz}$, are depicted in the table. Although the calculated results are closely related to the experimental observations, a larger van der Waals diameter for protons may be needed to achieve better agreements at the low temperatures studied.

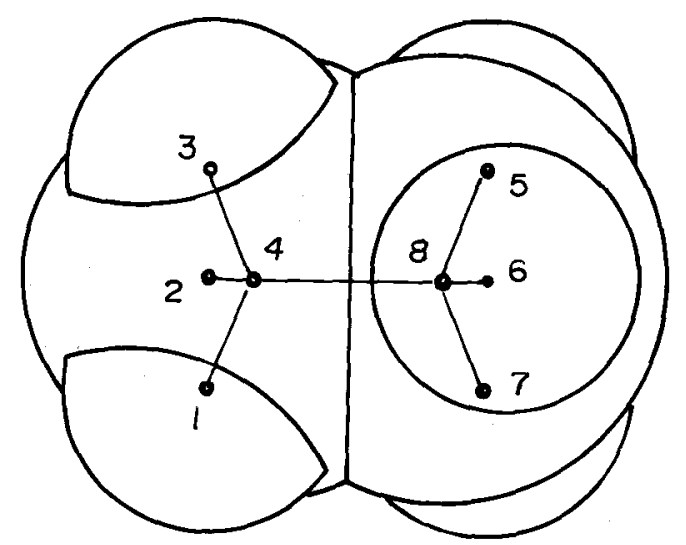

Figure 3. The RISM model for ethane. Atoms 1 to 3 and 5 to 7 are hydrogens, and 4 and 8 are carbons. The model parameters are given in the text. 
Comparison of experimental and calculated proton relaxation rates in liquid ethane at $90.71 \mathrm{~K}$.

\begin{tabular}{rccc}
\hline$v / \mathrm{MHz}$ & 3 & 30 & 73 \\
\hline$\left(1 / T_{1}\right) / \mathrm{s}^{-1}$ (expt.) & 0.502 & 0.458 & 0.430 \\
(calc.) & 0.555 & 0.527 & 0.502 \\
\hline
\end{tabular}

\subsection{Comparisons with a uniform pair distribution}

If a uniform distribution pcf is used we may simplify equation (1) and obtain the longitudinal relaxation rate in the motional narrowing region [3]

$$
1 / T_{1}=8 \pi n_{\mathrm{H}} \gamma_{\mathrm{H}}{ }^{4} h^{2} /(9 d D) \text {. }
$$

In liquid ethane and acetonitrile, the effects of invoking a uniform pair distribution gave 3 and 5 per cent enhancements in the respective relaxation rates in comparison with the results obtained using RISM pcfs, while in liquid chloroform the enhancement reaches 22 per cent. These may be explainẹd by the fact that there are six equivalent protons per ethane molecule and three equivalent protons per acetonitrile molecule. The $\mathrm{H}-\mathrm{H}$ pcfs in liquid ethane and/or in liquid acetonitrile have a larger magnitude than the one for liquid chloroform does within the distance of the first neighbouring proton sites (cf. figure 2). Also, the pcfs of the two former liquids behave similarly in this region. As a result, the incorporation of a uniform pcf have much more effect in the case of liquid chloroform. Thus, it may be noted that, for the nonuniform distribution of spin sites in a molecule, the pair-correlation effect becomes significant in spin relaxation.

To differentiate the contributions to the relaxation arising from the initial distribution and the derived effective force, we deliberately drop the force terms in the calculation. The result shows only a 2 per cent enhancement of relaxation rate for liquid chloroform in comparison with the calculation involving both terms for effective force and initial distribution. We then conclude that the initial distribution of spin sites determines the pair-correlation effect in spin relaxation processes.

\section{Conclusion}

We have shown in this work how the incorporation of pcfs derived from the RISM equation allow us to interpret proton relaxation rates in organic liquids. The approach consists basically, of considering the proton van der Waals diameter as the distance of closest approach between protons and the effects of the molecular pcf and its derived potential of average force on the equilibrium and dynamical distributions of protons in the liquids. However the orientational correlation has not been considered here. It may be treated by the method suggested by Zeidler [4] with the incorporation of the orientational correlation function determined from model calculations or experimental studies [16]. In addition, with the substitution of the proper functional forms discussed previously [3] for spherical Bessel functions, the correction of intermolecular impenetrability may thus be included. 
We thank the National Science Council of the Republic of China for support of this work.

\section{REFERENCES}

[1] Abragam, A., 1961, The Principle of Nuclear Magnetism (Oxford U.P.), Chap. VIII.

[2] Hubbard, P. S., 1963, Phys. Rev., 131, 275.

[3] Hwang, L-P., and Freed, J. H., 1975, F. chem. Phys., 63, 4017.

[4] Zeidler, M. D., 1975, Molec. Phys., 30, 1441.

[5] Albrand, J. P., Taieb, P. H., Ries, P. H., and Belorizky, E., 1983, F. chem. Phys., 78, 5809.

[6] Hsu, C. S., Chandler, D., and Lowden, L. J., 1976, Chem. Phys., 14, 213.

[7] Hsu, C. S., and Chandler, D., 1978, Molec. Phys., 36, 215.

[8] Hsu, C. S., and Chandler, D., 1979, Molec. Phys., 37, 299.

[9] (a) Chander, D., 1982, The Liquid State of Matter, edited by E. W. Montroll and J. L. Lebowitz (North-Holland Publishing Co.) ; (b) 1978, A. Rev. phys. Chem., 29, 441 , and references therein.

[10] LOWDEN, L. J., RISM, RISMGR, RISMSK : PROGRAMME NUMBER QCPE 306 ; Quantum Chemistry Computer Exchange, Indiana University, Bloomington, Indiana 47401, U.S.A.

[11] Pauling, L., 1960, The Nature of the Chemical Bond, 3rd edition (Cornell University Press).

[12] ZeldLer, M. D., 1971, Ber. Bunsenges. phys. Chem., 75, 769.

[13] Woessner, D. E., SNowden, B. S., Jr., and Strom, E. E., 1968, Molec. Phys., 14, 265.

[14] Bender, H. J., and Zeidler, M. D., 1971, Ber. Bunsenges. phys. Chem., 75, 236.

[15] Harmon, J. F., and Muller, B. H., 1969, Phys. Rev., 182, 400.

[16] Bertagnoli. , H., 1981, Ber. Bunsenges. phys. Chem., 85, 644. 